\title{
Electromyography in Front Crawl Technique - Case Study
}

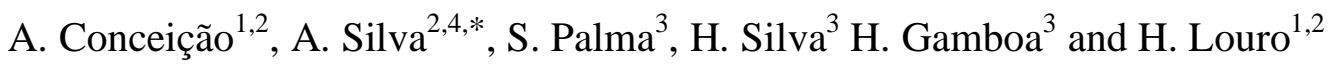 \\ ${ }^{1}$ Sports Sciences School of Rio Maior, Polytechnic Institute of Santarém, Portugal \\ ${ }^{2}$ Research Center for Sport, Health and Human Development (CIDESD), UTAD, Vila Real, Portugal \\ ${ }^{3}$ PLUX - Wireless Biosignals, Lisboa Portugal \\ ${ }^{4}$ Department of C. of Sport, Exercise and Health of University of Trás-os-Montes and Alto Douro; Vila Real, Portugal
}

\begin{abstract}
The need to develop systems electromyographic (EMG) in the aquatic environment, has led several researchers to refine these instruments to ensure the credibility of the data provided by EMG. The aim of this study is to characterize the behavior of two muscle muscles involved in the crawl technique (biceps brachii and triceps brachii) over a test of $200 \mathrm{~m}$ crawl.

1 male swimmer trained was subjected to a test consisting of a maximum voluntary contraction (MVC) of the Biceps Brachii $(B B)$ and Triceps Brachii $(T B)$ for standardization. A protocol of $4 \times 50 \mathrm{~m}$ with an interval of 15 seconds at a swimming speed of pre-established, making each part to $95 \%$ of transit time for $200 \mathrm{~m}$ crawl. EMG surface electrodes were used with a Wirelless bioPLUX.research. The EMG signal of the BB and TB muscles of the right arm was removed throughout the test and then synchronized with the video image, and selected 5 cycles of swimming on all identical pathways.

There is a gradual decrease of its average muscle activity. The BB was about $43 \%$ and TB was $26 \%$. The largest variation on it, in the case of $\mathrm{BB}$, between the third and fourth route $(21 \%)$, and in the case of $\mathrm{TB}$, between the second and third route $(14 \%)$.

The muscles studied demonstrated changes in the duration of swim cycles, indicating that, there is a decrease in muscle activity, this supposed to be due to limitations in production capacity under swimmer and the characteristics of the art of swimming which are changing as increasing fatigue.
\end{abstract}

Keywords: Electromyography, kinematics analysis, front crawl technique.

\section{INTRODUCTION}

It is important to find practical solutions that can help the swimmers and their coaches during the training process in order to better its performance. The front crawl technique gives a propulsive efficiency slightly above 50\% [1] because the position of the body which is inherent and can travel underwater oriented, creating from propellant with close direction and sense of direction of displacement of the body [2]. The efficiency of the arm is critical to the success of the athlete [3].

The need to develop systems electromyographic (EMG) to work in the aquatic environment, has led several researchers $[4,5]$ to refine these instruments to ensure the credibility of the data provided by EMG, both to the pattern of muscle activation [6-10], the muscle involvement and its variations in various technical swimming [11], the amendments to the EMG signal during maximal tests [12].

*Address correspondence to this author at the Department of Sport Sciences, University of Trás-os-Montes and Alto Douro, Vila Real, Portugal, Rua Dr. Manuel Cardona, 5000 Vila Real Portugal;

Tel: +351 259350000; E-mail: Ajsilva@utad.pt
The aim of this study is to characterize the behavior of two muscle muscles involved in the crawl technique (biceps brachii and triceps brachii) over a proof of $200 \mathrm{~m}$ crawl.

\section{METHODS}

One male swimmer trained, with 20 years old, $72 \mathrm{~kg}, 1.77$ meters, $1.78 \mathrm{~cm}$ in size and with a \%Fat mass: 6.6 was subjected to a test consisting of a maximum voluntary contraction (MVC) of the Biceps Brachii (BB) and Triceps brachii $(T B)$ for standardization. After this process the swimmer made a experimental protocol of $4 \times 50 \mathrm{~m}$ in the crawl technique with an interval of 15 seconds at a swimming speed of pre-established, making each part to $95 \%$ of transit time for $200 \mathrm{~m}$ crawl. In order to collect the EMG signal form the water proof surface electrodes were used with the Wireless bioPLUX.research system. The EMG signal of the BB and TB muscles of the right arm was collected throughout the test and then synchronized with the video image using a led marker collected in both systems. We selected 5 cycles of swimming on all identical pathways $(15 \mathrm{~m})$ and filtered using a low pass filter implemented using a sliding window ("smooth") of 300 points. The signal was normalized based on the maximum voluntary contraction measured on the signal of the pre-test for each muscle. The signal processing 
was done offline using the numpy toolbox (version 1.0) in the python language (version 2.4).

\section{RESULTS}

Having determined the moments of Entry and Exit of the hand in the water based on the sign of acceleration, we determined the length of the swim cycles during the test. In Table 1, we present the duration (measured from the stages of entry and exit of the hand in water) and we conclude that it increases gradually over the four paths of $50 \mathrm{~m}$. Between the beginning and end of the test, the average length of cycles increased from $0.87 \mathrm{~s}$ to 1:07 $\mathrm{s}$, consisting in a relative increase of $23 \%$ with the largest relative increase (12\%) occurred between the first and second path.

The test results are presented in Table $\mathbf{2}$, in absolute and relative values of the EMG mean \pm standard deviation over the test. Comparing the average values of EMG of BB and TB over the four pathways to swim $(4 \mathrm{x} 50 \mathrm{~m})$, the results shows that there is a gradual decrease in the average muscle activity. Between the beginning and end of the test, the relative decrease of the $\mathrm{BB}$ was $43 \%$, while on the $\mathrm{TB}$ muscle the decrease was $26 \%$. The largest variation in the case of $\mathrm{BB}$, between the third and fourth route was $21 \%$ ) and in the case of TB, between the second and third route was $14 \%$.

Representing the average EMG recorded for each cycle of swimming in terms of its duration (Fig. 1), we conclude that when the cycles are longer, the average muscle activity tends to be smaller. When doing a statistical analysis the variables correlate moderately. This trend is found both in $\mathrm{BB}(\mathrm{r}=-0.6)$ and in TB $(\mathrm{r}=-0.6)$

\section{DISCUSSION}

The results of this case study indicate that both muscles have changes in the duration of swim cycles, pointing that, with increasing duration of cycles of swimming, there is a decrease in muscle activity. This is supposed to be caused by limitations in production capacity of the swimmer and/or due to characteristics of the art of swimming which are changing

Table 1. Average Durantion \pm Standard Deviation of the Cycle of Swimming in Each Part of Swimming Throught the Test

\begin{tabular}{|l|c|c|c|}
\hline & 1st_50m & 2nd_50m & 3thr_50m \\
\hline \hline Mean \pm DV (s) & $0.87 \pm 0.10$ & $0.98 \pm 0.14$ & $1.07 \pm 0.12$ \\
\hline$\%$ of mean duration of 1st route & 100 & 112.6 & 123 \\
\hline$\%$ of mean duration of last route & -- & 112.6 & 109.2 \\
\hline
\end{tabular}

Table 2. Muscle Activity (\% MVC) Average of the Monitored Muscles and Their Variations of Each Part of Swimming

\begin{tabular}{|c|c|c|c|c|}
\hline & $50 \mathrm{~m}$ & $50 \mathrm{~m}$ & $50 \mathrm{~m}$ & $50 \mathrm{~m}$ \\
\hline \multicolumn{5}{|l|}{ Biceps Brachii(BB) } \\
\hline Mean \pm DV $(\%$ CVM $)$ & $25,68 \pm 16,09$ & $20,6 \pm 13,95$ & $18,39 \pm 13,8$ & $14,58 \pm 12,19$ \\
\hline$\%$ EMG of 1 st route & 100 & 80.2 & 71.6 & 56.8 \\
\hline$\%$ EMG of last route & -- & 80.2 & 89.3 & 79.3 \\
\hline \multicolumn{5}{|l|}{ Triceps Brachii $(B B)$} \\
\hline Mean \pm DV $(\%$ CVM $)$ & $52,84 \pm 36,97$ & $46,2 \pm 30,57$ & $39,52 \pm 28,14$ & $39,32 \pm 28$ \\
\hline$\%$ EMG of 1 st route & 100 & 87.4 & 74.8 & 74.4 \\
\hline$\%$ EMG of last route & -- & 87.4 & 85.5 & 99.5 \\
\hline
\end{tabular}

$\mathrm{A}(\mathrm{BB})$

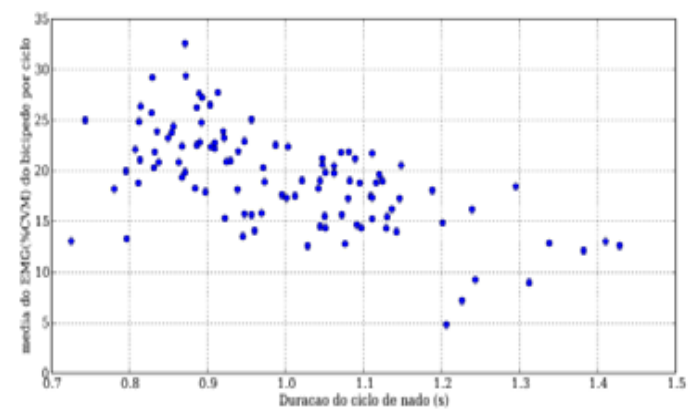

$\mathrm{B}(\mathrm{TB})$

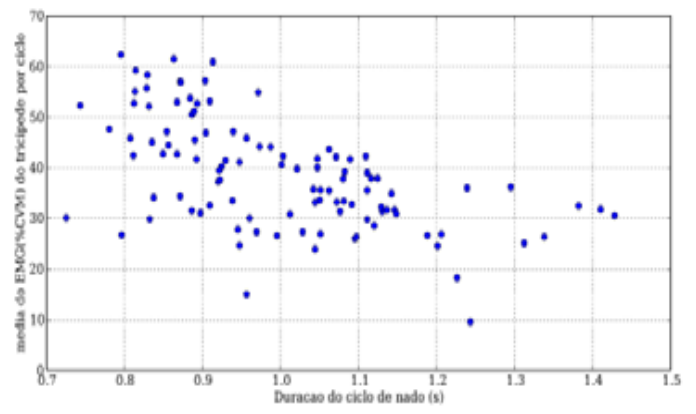

Fig. (1). Relationship between the duration of cycle of swimming activity and mean biceps brachii muscle (A) and tricpes brachii (B). 
with fatigue increase. As found during the course increased the duration of the slide, making the swimmer less mechanic efficient, according to the values of muscle activation observed.

The coach must use a planning strategy towards the work of resistance and / or strength taking into account the process observed in the current experiment.

\section{REFERENCES}

[1] Toussaint HM. Mechanics an energetics of swimming. Amesterdam: published by the author 1998 .

[2] Alves F. Swimming economy and provide competitive mechanical and metabolic determinants in alternating techniques.PHD thesis (not published). UTL-FMH. Lisboa 1995.

[3] Skinner J. Distance per stroke and time. Swimm Technol 1997; 34: 37-9.

[4] Gonçalves P, Pereira S, Vilar S, et al. Underwater Electromyograph system and His dialog with other instrumentation. Book of Abstracts- X International Symposium Biomechanics and Medicine in Swimming. Porto. Portugal 2006.

[5] Möller E, Krumholz F, Black G, et al. EMG Analysis of the muscles pectoralis major and deltoid posterior. Book of Abstracts$\mathrm{X}$ International Symposium Biomechanics and Medicine in Swimming. Porto. Portugal 2006.
Clarys J. A review of EMG in swimming: explanation of facts and/ or feedback information. In: Hollander A, Huijing P, Groot G, Eds. Biomechanics and medicine in swimming. Champaign, Illinois, USA: Human Kinetics Publisher 1983; pp. 123-35.

[7] Pink M, Perry J, Browne A, Scovazzo ML, Kerrigan J. The normal shoulder during freestyle swimming. An electromyographic and cinematographic analysis of twelve muscles. Am J Sports Med 1991; 19 (6): 569-76.

[8] Rouard A, Billat R, Deschodt V, Clarys J. Muscular activation in sweep movements of the upper limb in freestyle swimming. In: Riehle H, Vieten M, Eds. XIX I.S.B. Congress 1993; pp. 781-82.

[9] Caty V, Aujouannet Y, Hintzy F, et al. Wrist stabilisation and forearm muscle coactivation during freestyle swimming. J Electromyograp Kinesiol 2007; 17(3): 285-91

[10] Figueiredo, P, Sousa, A, Pereira, S, et al. Estudo Electromiográfico do músculo deltóide durante arecuperação dos membros superiores na técnica de crol. Efdeportes.n.113 2007.

[11] Wakayoshi K, Moritani T, Mutoh Y, et al. Electromyography evidence of selective muscle fatigue during competitive swimming. Med Sci Aquat Sports 1994; 39: 16-23.

[12] Stirn I, Vizintin T, Kapus V, Jarm T, Strojnik V. Change of mean frequency of EMG signal during 100 meter maximal free style swimming. Book Chapter 11th Mediterranean Conference on Medical and Biomedical Engineering and Computing Ljubljana, Slovenia 2007.

(C) Conceição et al.; Licensee Bentham Open.

This is an open access article licensed under the terms of the Creative Commons Attribution Non-Commercial License (http://creativecommons.org/licenses/by-nc/3.0/) which permits unrestricted, non-commercial use, distribution and reproduction in any medium, provided the work is properly cited. 